AIRWAY BIOLOGY

\title{
Protective role of metallothionein in acute lung injury induced by bacterial endotoxin
}

\author{
H Takano, K Inoue, R Yanagisawa, M Sato, A Shimada, T Morita, M Sawada, K Nakamura, \\ C Sanbongi, T Yoshikawa
}

Thorax 2004;59:1057-1062. doi: 10.1136/thx.2004.024232

See end of article for authors' affiliations

Correspondence to:

Dr H Takano,

Pathophysiology Research Team, National Institute for Environmental Studies,

16-2 Onogawa, Tsukuba 305-0053, Japan;

htakano@nies.go.jp

Received 3 March 2004 Accepted 19 July 2004
Background: Metallothionein (MT) is a protein that can be induced by inflammatory mediators and participate in cytoprotection. However, its role in inflammation remains to be established. A study was undertaken to determine whether intrinsic MT protects against acute inflammatory lung injury induced by bacterial endotoxin in MT-I/II knock out (-/-) and wild type (WT) mice.

Methods: MT (-/-) and WT mice were given vehicle or lipopolysaccharide (LPS, $125 \mu \mathrm{g} / \mathrm{kg}$ ) intratracheally and the cellular profile of the bronchoalveolar lavage (BAL) fluid, pulmonary oedema, lung histology, expression of proinflammatory molecules, and nuclear localisation of nuclear factor- $\kappa B(N F-\kappa B)$ in the lung were evaluated.

Results: MT (-/-) mice were more susceptible than WT mice to lung inflammation, especially to lung oedema induced by intratracheal challenge with LPS. After LPS challenge, MT deficiency enhanced vacuolar degeneration of pulmonary endothelial cells and type I alveolar epithelial cells and caused focal loss of the basement membrane. LPS treatment caused no significant differences in the enhanced expression of proinflammatory cytokines and chemokines nor in the activation of the NF- $\kappa$ B pathway in the lung between the two genotypes. Lipid peroxide levels in the lungs were significantly higher in LPS treated MT (-/-) mice than in LPS treated WT mice.

Conclusions: Endogenous MT protects against acute lung injury related to LPS. The effects are possibly mediated by the enhancement of pulmonary endothelial and epithelial integrity, not by the inhibition of the NF- $\kappa$ B pathway.
M etallothionein (MT) is a highly conserved, low molecular weight, cysteine rich protein. Since cysteine residues of MT bind and store metal ions, it has been proposed that MT may have an important role in homeostasis and detoxication of heavy metals. ${ }^{1}$ It can react also with free radicals and electrophils because of its high sulfhydryl content, ${ }^{2{ }^{3}}$ and can serve as a sacrificial scavenger for hydroxyl radicals in vitro ${ }^{4}$ and also protects against free radical induced DNA damage. ${ }^{5-7}$ In addition, MT is induced by heavy metals or oxidative stress producing chemicals, ${ }^{8}$ and exhibits cytoprotection against toxicity of heavy metals or alkylating anticancer drugs ${ }^{3}$ as well as against oxidative stress related organ damage. ${ }^{9}{ }^{10}$ MT- $I$ and MT-II genes are constitutively expressed in the liver and are highly induced by metals, glucocorticoids, and inflammatory mediators. Proinflammatory cytokines, including tumour necrosis factor (TNF)- $\alpha$, interleukin (IL)- 1 , IL- 6 , and interferon- $\gamma$, induce hepatic MT gene expression in vivo. However, there are conflicting reports about the role of MT in inflammatory processes. In brief, MT plays a pivotal role in mediating the harmful effects of TNF induced shock ${ }^{11}$ but MT (-/-) mice have been reported to be more sensitive to lipopolysaccharide (LPS) induced lethal shock. ${ }^{12}$

Acute lung injury is characterised by neutrophilic inflammation, increased expression of proinflammatory cytokines, loss of epithelial and endothelial integrity, and the development of interstitial oedema. ${ }^{13-16}$ Intratracheal instillation of LPS produces a well recognised model of acute lung injury leading to the activation of alveolar macroghages, tissue infiltration of neutrophils, and interstitial oedema. ${ }^{17}$ Although inhalation of LPS has been reported to induce MT gene and protein in the lung in vivo, ${ }^{18} 19$ there is no evidence for the direct contribution of MT in acute lung injury elicited by LPS.
The development of acute lung injury requires several pulmonary cell populations, transcriptional regulatory factors, and proinflammatory molecules. Nuclear factor- $\kappa \mathrm{B}(\mathrm{NF}-\kappa \mathrm{B})$ activation and the subsequent expression of proinflammatory mediators also have an important role. ${ }^{20}{ }^{21}$ However, the effects of MT on NF- $\kappa \mathrm{B}$ activation is uncertain. ${ }^{22}{ }^{23}$

This study was undertaken to determine whether intrinsic MT has a role in protecting against acute lung injury induced by LPS using MT-I/II null (MT $(-/-)$ ) mice and control wild type (WT) mice. The mechanisms by which MT protects against acute lung injury were also studied and its role in the $\mathrm{NF}-\kappa \mathrm{B}$ pathway in vivo determined.

\section{METHODS}

Animals and study protocol

MT (-/-) mice whose MT-I and MT-II genes had null mutation and WT mice were provided by Dr Choo (Murdoch Institute for Research into Birth Defects, Royal Children's Hospital, Australia). ${ }^{24}$ They were of a mixed genetic background of 129 Ola and C57BL/6 strains. F1 hybrid mice were mated with $\mathrm{C} 57 \mathrm{BL} / 6$ mice and their offspring were backcrossed to C57BL/6 for six generations in the National Institute for Environmental Studies (NIES; Tsukuba, Japan), reproducing normally and displaying no overt abnormality in physical state and behaviour. These mice were routinely bled in the vivarium of NIES as previously described. ${ }^{10}$

MT (-/-) and WT mice were treated with vehicle or LPS ( $E$ coli B55:05, Difco Lab, Detroit, MI, USA). For both

Abbreviations: IL, interleukin; KC, keratinocyte chemoattractant; LPS, lipopolysaccharide; MCP-1, macrophage chemoattractant protein; MIP$1 \alpha$, macrophage inflammatory protein-1 1 ; MT, metallothionein; NF- $\mathrm{\kappa B}$, nuclear factor- $\mathrm{\kappa} B$ 
genotypes the vehicle groups received $100 \mu$ phosphate buffered saline (PBS) at pH 7.4 (Nissui Pharmaceutical Co, Tokyo, Japan) by intratracheal inoculation. The LPS groups received $125 \mu \mathrm{g} / \mathrm{kg}$ LPS dissolved in $100 \mu \mathrm{l}$ of the same vehicle. Intratracheal inoculation was conducted as described previously. ${ }^{25} 26$

Lung water content, bronchoalveolar lavage (BAL), and measurement of cytokines and lipid peroxides The lungs were weighed and dried as previously reported. ${ }^{27}$ The wet lung weight - dry lung weight/body weight was calculated ( $\mathrm{n}=5$ in each group). BAL and cell counts were conducted as previously described ( $n=5$ in each group). ${ }^{26}{ }^{28}$ Enzyme linked immunosorbent assays (ELISA) for IL- $1 \beta$ (Endogen, Cambridge, MA, USA), macrophage inflammatory protein (MIP)- $1 \alpha$ (R\&D Systems, Minneapolis, MN, USA), macrophage chemoattractant protein (MCP)-1 (R\&D Systems), and keratinocyte chemoattractant (KC) (R\&D Systems) in the lung tissue supernatants $(n=8$ in each group) were carried out as reported elsewhere. ${ }^{26}{ }^{28}$ Lipid peroxide in the lung was measured by a Determiner LPO kit (Kyowa Medics Co Ltd, Tokyo, Japan) ( $\mathrm{n}=8$ in each group).

\section{Microscopic examination}

The lungs were fixed and stained with haematoxylin and eosin as previously described $\left(\mathrm{n}=4\right.$ in each group). ${ }^{25}$ Lung tissue was fixed with $2.5 \%$ glutaraldehyde and rinsed in $0.1 \mathrm{M}$ phosphate buffer ( $\mathrm{pH} 7.4 ; \mathrm{n}=4$ in each group). Ultrathin sections were stained with uranyl acetate and lead citrate and examined with a JEM-100CX electron microscope (JEOL, Tokyo, Japan).

\section{Preparation of nuclear and cytoplasmic protein and Western blot analysis}

Nuclear protein extracts $(\mathrm{n}=5$ in each group) were examined by Western blot analysis using a rabbit anti-p65 antibody (Santa Cruz Biotechnology, Santa Cruz, CA, USA) and the bands were quantitated as described previously. ${ }^{26}$

\section{Statistical analysis}

Data were reported as mean (SE) values except for the cellular profiles of BAL fluid and the lung water content using Stat View version 4.0 (Abacus Concepts Inc, Berkeley, CA, USA) as previously described..$^{25}$ Differences in cellular profiles of BAL fluid, lung water content, and nuclear localisation of p65 subunit of $\mathrm{NF}-\kappa \mathrm{B}$ were analysed by a Kruskal-Wallis test followed by a Mann-Whitney U test using

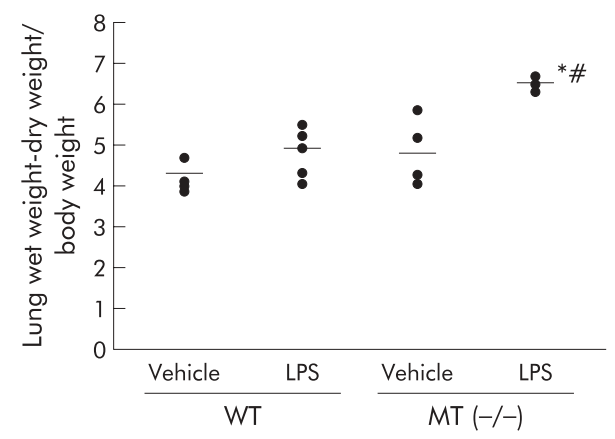

Figure 1 Lung water content in wild type (WT) and metallothionein (MT)-I/II knock out (-/-) mice challenged with lipopolysaccharide (LPS, $125 \mu \mathrm{g} / \mathrm{kg}$ ). The bilateral lungs were weighed immediately after exsanguination 24 hours following the intratracheal administration of LPS or vehicle, dried in an oven at $95^{\circ} \mathrm{C}$ for 48 hours, and the wet lung weight - the dry lung weight/body weight was calculated. The results are shown as mean (SE) values $(n=5)$. ${ }^{*} p<0.01 v$ vehicle treated mice; $\# p<0.05 \vee$ LPS treated WT mice.
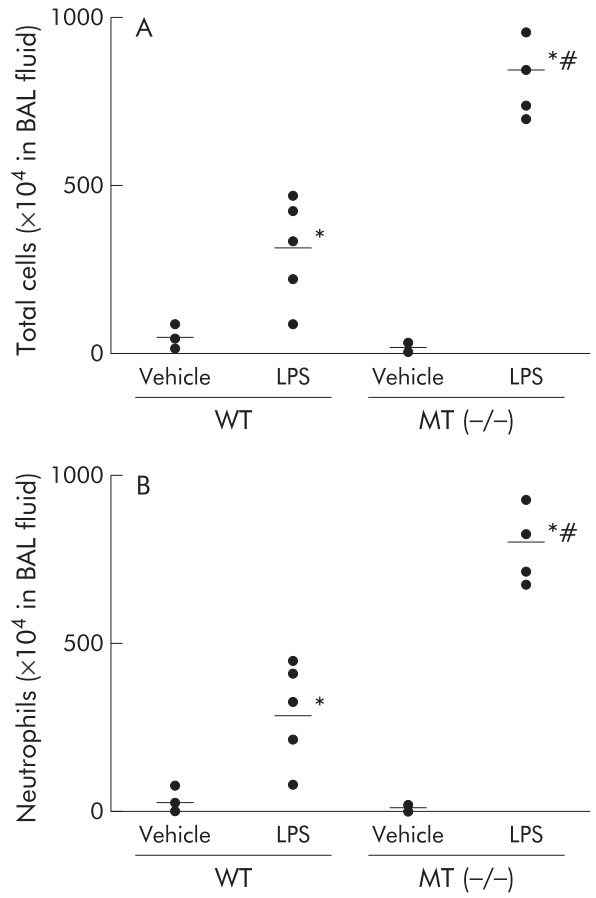

Figure 2 (A) Total cells and (B) neutrophils in bronchoalveolar lavage (BAL) fluid in WT and MT $(-/-)$ mice challenged with LPS. Twenty four hours after the intratracheal administration of vehicle or LPS the lungs were lavaged for the analysis of BAL fluid. The total cell count was determined on a fresh fluid specimen using a haemocytometer. Differential cell counts were assessed on cytological preparations stained with Diff-Quik. The results are shown as mean (SE) values $(n=5)$. ${ }^{*} \mathrm{p}<0.05 v$ vehicle treated mice; \#p<0.05 $v$ LPS treated WT mice.

the SPSS 8.0 statistical package for Windows (Chicago, IL, USA). Differences in other data were analysed by ANOVA followed by Fisher's PLSD test (Stat View version 4.0). $\mathrm{p}$ values of $<0.05$ were considered statistically significant.

\section{RESULTS}

\section{Role of MT in acute lung injury induced by bacterial endotoxin}

To examine the role of MT in pulmonary oedema related to bacterial endotoxin we measured the lung water content 24 hours after the intratracheal instillation of vehicle or LPS (fig 1). LPS increased the lung water content in both genotypes of mice compared with the vehicle $(p<0.01$ for MT $(-/-)$ mice, not significant in WT mice). Following treatment with LPS, MT (-/-) mice had a significant and marked increase in the lung water content compared with WT mice $(\mathrm{p}<0.05)$.

To determine the role of MT in neutrophilic lung inflammation induced by bacterial endotoxin, the cellular profile of BAL fluid 24 hours after intratracheal instillation was examined. In both genotypes of mice LPS treatment induced significant increases in the numbers of total cells (fig 2A) and neutrophils (fig 2B) compared with vehicle treatment $(p<0.05)$. LPS treatment caused greater and significant increases in the number of total cells (fig 2A) and neutrophils (fig 2B) in the BAL fluid in MT $(-/-)$ mice than in WT mice $(\mathrm{p}<0.05)$.

\section{Effect of MT deficiency on LPS induced histopathological and ultrastructural changes in the lung}

To determine the differences in the histological changes after LPS treatment in the presence or absence of MT, lung 

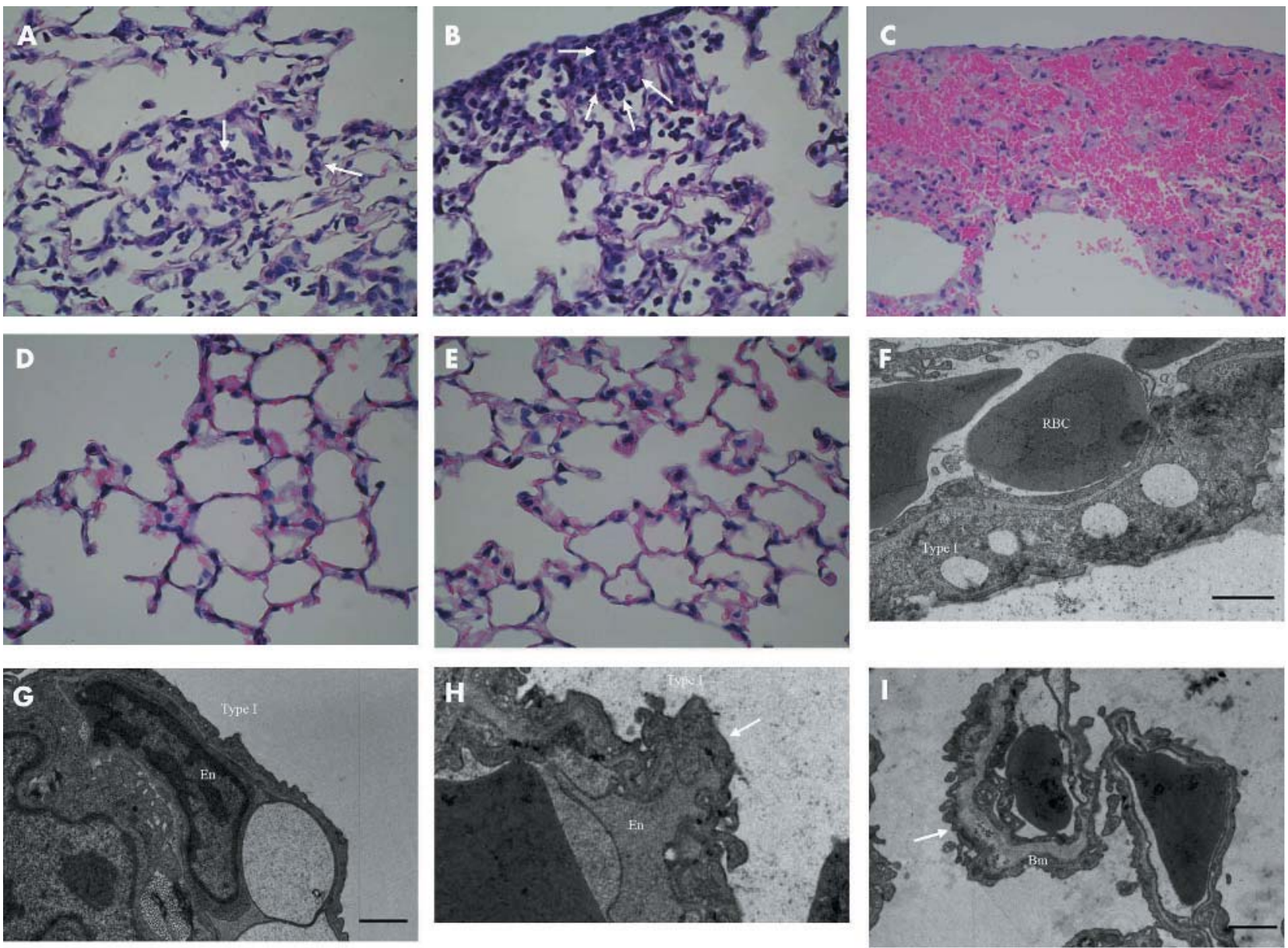

Figure 3 LPS induced histopathological changes (A-E) and ultrastructural changes (F-I) in the lungs of WT and MT (-/ -) mice. WT mice: (A) LPS, (D) vehicle; MT (-/-) mice: (B, C) LPS, (E) vehicle. The histopathological changes induced by LPS in WT mice showed moderate infiltration of neutrophils (A; arrows). LPS treatment showed more significant damage in the lungs of MT (-/ ) mice which included recruitment of neutrophils (B; arrows) and alveolar haemorrhage (C). Original magnification $\times 320(A, B, D, E), \times 250$ (C). LPS induced ultrastructural changes included the thickening of the alveolar basement membrane (arrow) in WT mice (I). LPS treated MT (-/-) mice had large vacuoles in the cytoplasm of endothelial cells and type alveolar epithelial cells $(F, G)$. Focal loss of the basement membrane (arrow) was seen in the alveolar walls of LPS challenged MT $(-/-)$ mice (H). RBC, red blood cell; type I, type I alveolar epithelial cell; En, endothelial cell; Bm, basement membrane. Bar $=1 \mu \mathrm{m}$.
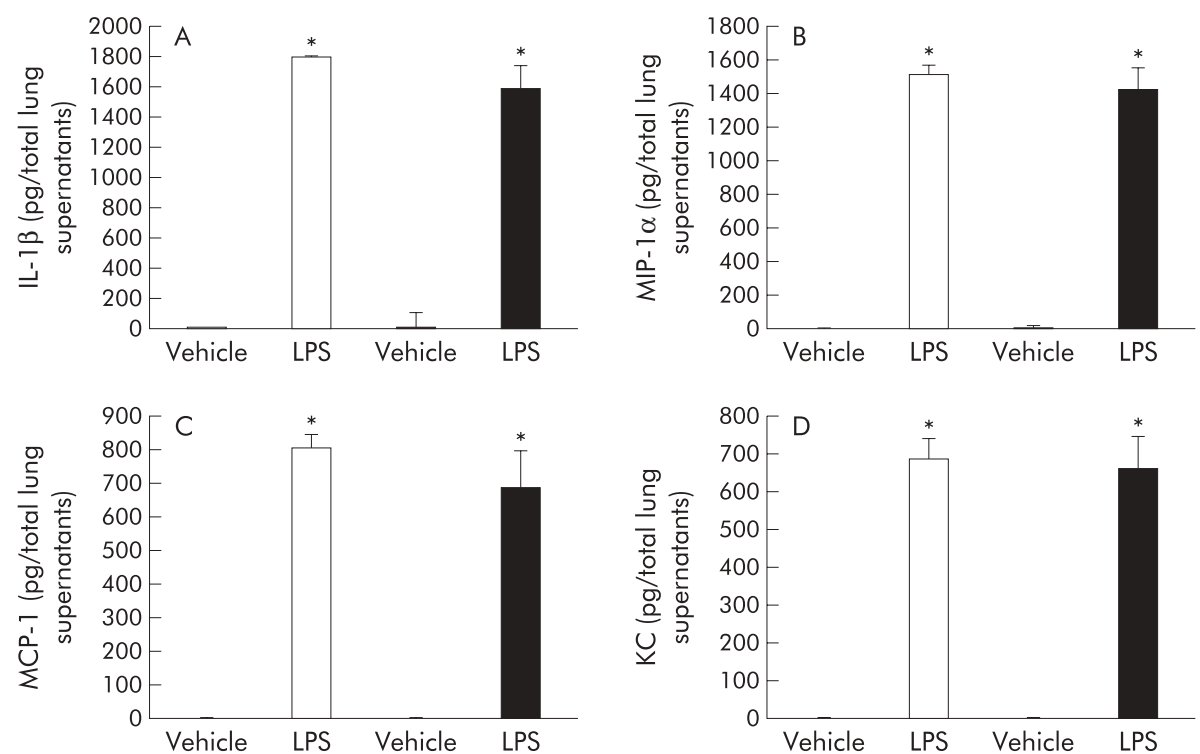

Figure 4 Protein levels of (A) interleukin (IL)-1 $\beta$, (B) macrophage inflammatory protein (MIP)-1 $\alpha$, (C) macrophage chemoattractant protein (MCP)-1, and (D) keratinocyte chemoattractant (KC) in lung tissue supernatants 24 hours after challenge with vehicle or LPS ( $n=8$ in each group) measured by enzyme linked immunosorbent assays. White bar, WT mice; black bar, MT $(-/-)$ mice. Results are mean (SE) values. * $p<0.05 v$ vehicle treated mice. 


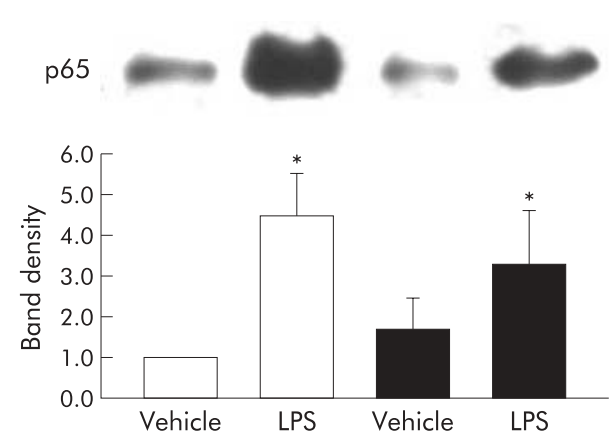

Figure 5 Nuclear localisation of the $\mathrm{p} 65$ subunit of nuclear factor- $\mathrm{kB}$ (NF- $\kappa B)$ in WT and MT $(-/-)$ mice treated with vehicle or LPS. Nuclear localisation of the $\mathrm{p} 65$ subunit of NF- $\mathrm{KB}$ was investigated 2 hours after intratracheal administration using Western blot analysis. The top panel shows the actual membrane pictures of $\mathrm{p} 65$ and the bottom panel shows the band density for p65. White bar, WT mice; black bar, MT (-/-) mice. Each density represents the mean (SE) of at least five animals per group. ${ }^{*} p<0.05 v$ vehicle treated mice.

specimens stained with haematoxylin and eosin were examined 24 hours after intratracheal instillation. In the presence of LPS, WT mice showed a moderate infiltration of neutrophils (fig 3A) while in MT $(-/-)$ mice there was a marked recruitment of neutrophils and interstitial oedema with alveolar haemorrhage (fig 3B, C). Vehicle administration alone caused no histological changes in either genotype (fig 3D, E).

To elucidate the mechanisms by which MT protects against acute lung injury, especially against aggravated vascular permeability related to bacterial endotoxin, the ultrastructural changes in the lung were examined by electron microscopy 24 hours after intratracheal instillation. LPS treated MT (-/-) mice had large vacuoles in the cytoplasm of the endothelial cells and type I alveolar epithelial cells (fig 3F, G) accompanied by a focal loss of basement membrane in the alveolar walls (fig $3 \mathrm{H}$ ). In LPS treated WT mice, on the other hand, only thickening of the alveolar basement membrane occurred (fig 3I). Following vehicle the alveolar wall was normal in both MT $(-/-)$ and WT mice (data not shown).

\section{Effect of MT deficiency on lung expression of proinflammatory cytokines and chemokines related to bacterial endotoxin}

To investigate the role of MT in the lung expression of proinflammatory cytokines and chemokines related to bacterial endotoxin, we compared the protein levels of IL$1 \beta$, MIP- $1 \alpha$, MCP- 1 , and KC in lung tissue supernatants from the four experimental groups 24 hours after intratracheal instillation. LPS treatment induced significant increases in these cytokines and chemokines compared with vehicle treatment in both genotypes (fig $4 \mathrm{~A}-\mathrm{D}, \mathrm{p}<0.01$ ). In the presence of LPS treatment, however, the local expression of these cytokines and chemokines was not significantly different between the two genotypes (fig 4A-D).

\section{Effect of MT deficiency on NF- $\kappa$ B activation related to bacterial endotoxin}

To determine whether the absence of MT affects the activation of NF- $\kappa \mathrm{B}$ related to bacterial endotoxin in vivo, we compared nuclear protein levels of the p65 subunit of NF$\kappa \mathrm{B}$ in the lungs 2 hours following intratracheal instillation. In both genotypes LPS treatment significantly increased nuclear localisation of the p65 subunit of NF- $\mathrm{\kappa B}$ compared with vehicle treatment (fig $5, \mathrm{p}<0.05$ ). However, there were

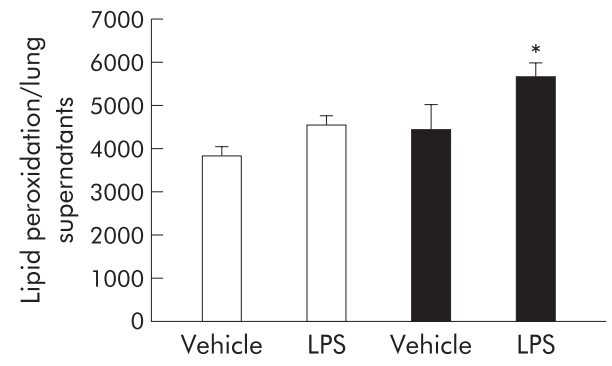

Figure 6 Formation of lipid peroxides in lung tissue supernatants determined by Determiner LPO kit. Values are mean (SE) of six animals per group. White bar, WT mice; black bar, MT (-/-) mice. * $p<0.05 v$ other groups.

no significant differences between the protein levels of MT $(-/-)$ mice and WT mice after LPS administration (fig 5).

\section{Effects of MT on lipid peroxidation induced by bacterial endotoxin}

We also examined the effects of MT on lipid peroxide formation in the lung. Compared with the vehicle, LPS increased the lipid peroxide content in both genotypes of mice (fig 6; $\mathrm{p}<0.05$ for MT $(-/-)$ mice, difference not significant in WT mice). LPS treatment led to a significantly greater increase in the lipid peroxide content in MT $(-/-)$ mice than in WT mice (fig 6; $\mathrm{p}<0.05$ ).

\section{DISCUSSION}

This study has shown that MT $(-/-)$ mice are more sensitive to acute lung injury induced by intratracheal administration of LPS than WT mice, especially to pulmonary oedema. Electroscopic examination showed that LPS treatment causes vacuolar degeneration of pulmonary vascular endothelial cells and type I alveolar epithelial cells, and focal loss of the basement membrane in MT $(-/-)$ mice but not in WT mice. The local expression of proinflammatory cytokines and chemokines and the activation of the NF- $\mathrm{KB}$ pathway in the lung induced by LPS were not significantly different in the presence or absence of MT. The lipid peroxide content in the lungs was significantly higher in LPS treated MT $(-/-)$ mice than in LPS treated WT mice.

MT is a regulator of zinc and copper homeostasis. It has recently been reported that several inflammatory stimuli induce hepatic MT. The induction of MT is mediated by several cytokines such as IL-1, IL-6, TNF- $\alpha$, and interferon$\gamma \cdot{ }^{1929-33}$ More recently, it has been reported that MT $(-/-)$ mice are protected from TNF induced lethal shock compared with WT mice. ${ }^{11}$ In addition, MT- $I$ overexpressing mice are more sensitive to the lethal effects of TNF than WT mice. ${ }^{11}$ In contrast, Kimura and colleagues have reported that MT (-/-) mice are more sensitive to LPS induced lethal shock in GalN sensitised mice through the reduction of $\alpha_{1}$-acid glycoprotein. ${ }^{12}$ The specific role of MT in inflammation therefore remains controversial.

As for lung inflammation, inhaled LPS induces MT expression in vivo, ${ }^{18}{ }^{19}$ which suggests that MT may play a role in lung inflammation induced by LPS. In particular, LPS induces MT in epithelial cells in the lung. ${ }^{18}$ We also confirmed that immunoreactive MT proteins in the lungs are found in the endothelial cells and alveolar epithelial cells of WT mice but not in those of MT $(-/-)$ mice (data not shown). In this study we found that MT $(-/-)$ mice were more susceptible to acute inflammatory lung injury induced by LPS than WT mice. In particular, pulmonary oedema induced by LPS was markedly enhanced by MT deficiency. Based on previous reports $^{1819}$ and the results of this study, it is suggested that 
MT may have protective properties against acute inflammatory lung injury induced by bacterial endotoxin, predominantly by protecting the integrity of the pulmonary vascular system.

Despite the sensitivity of the vascular endothelium to both heavy metal toxicity and oxidative stress, ${ }^{34}{ }^{35}$ little is known about the role of MT in vascular endothelial/epithelial integrity. In human lungs immunoreactive MT is detected in pleural endothelial cells and basal cells from the bronchial epithelium. ${ }^{36}$ On the other hand, cultured sheep pulmonary artery endothelial cells which overexpress MT are resistant to pro-oxidant stimuli in a metal independent fashion. ${ }^{37}$ It has also been postulated that MT protects endothelial cells against oxidative stress with or without subsequent increased expression of cytokines, thrombin, and endothelin-l in human venous endothelial cells. ${ }^{38}$

In the present study, electron histological examination of the lungs showed that LPS caused more pronounced damage of the vascular endothelial cells, endothelial basement membrane, and alveolar epithelial cells in MT $(-/-)$ mice than in WT mice. It is likely that the protective role of MT in this model is mediated through the maintenance of endothelial and epithelial integrity. Since enhanced expressions of cyclo-oxygenase (COX)- $2^{39}$ and Rho kinase ${ }^{40}$ have been reported to play a role in endothelial retraction and permeability, we examined the local expression of these proteins by Western blotting (data not shown). In both genotypes LPS treatment increased COX-2 and Rho kinase proteins in the lung compared with vehicle treatment. However, there were no significant differences in the levels of these two proteins between MT $(-/-)$ and WT mice after LPS administration (data not shown). Further studies are needed to elucidate the molecular mechanism by which MT protects the vascular integrity.

Activation of transcription factors such as NF- $\mathrm{BB}$ and the subsequent production of proinflammatory mediators play a critical role in the development of acute lung injury. NF- $\kappa B$ activation in the lung after intratracheal instillation of LPS is correlated with cytokine mRNA expression and neutrophilic alveolitis, supporting the idea that $N F-\kappa B$ activation is a pivotal event in the generation of neutrophilic lung inflammation. ${ }^{41}$ IL- $1 \beta$, MIP- $1 \alpha$, MCP- 1 and IL- 8 have also been shown to participate in the pathogenesis of acute lung injury. ${ }^{42-45}$ Intriguingly, MT is observed in the nucleus and/or the cytoplasm of cells, ${ }^{46}$ suggesting that it can interact with nuclear transcription factors. In fact, TNF induced NF- $\kappa B$ activation in WT cells has been reported to be lower than that in MT $(-/-)$ cells. ${ }^{23}$ Transfection of the MT- $I$ gene to MT $(-/-)$ cells reduces $\mathrm{NF}-\mathrm{KB}$ activation by suppressing the degradation of $\mathrm{I}-\kappa \mathrm{B}$, which suggests that MT functions as a negative regulator of NF- $\mathrm{KB}$ activation. In contrast, AbdelMageed and Agrawal ${ }^{22}$ have shown that MT potentiates the activation of NF- $\kappa \mathrm{B}$, so the apparent correlation between MT and NF- $\mathrm{BB}$ has not been established. In the present study MT deficiency did not alter the nuclear localisation of NF- $\kappa \mathrm{B}$. Although the levels of $\mathrm{NF}-\mathrm{\kappa B}$ induced proinflammatory molecules were increased in the lungs after LPS challenge, the differences between MT $(-/-)$ and WT mice were not statistically significant. Our results indicate that the protective effect of MT on acute lung injury related to bacterial endotoxin may not be mediated via NF- $\kappa \mathrm{B}$ dependent pathways. Further studies are needed to elucidate the role of MT in NF- $\mathrm{KB}$ mediated gene expression in other experimental models.

We have previously confirmed the expression of proinflammatory cytokines and chemokines including IL-1 $\beta$, MIP-1 $\alpha$, MCP-1, and KC in the lung 24 hours after the intratracheal administration of $\mathrm{LPS}^{26}{ }^{28}$ and the localisation of NF- $\kappa \mathrm{B} 2$ hours after LPS administration. ${ }^{26}$ Close correlations were found between NF- $\kappa$ B activation, the enhanced local expression of proinflammatory mediators, and the magnitude of acute lung injury. ${ }^{26}$ We therefore examined the expression of proinflammatory mediators 24 hours after intratracheal LPS challenge and the activation of NF- $\mathrm{KB} 2$ hours after LPS challenge in the present study. In addition, the overall trends for the expression of these proinflammatory proteins in the lung at other time points ( 2 and 6 hours after challenge) were similar to those at 24 hours after the intratracheal challenge in the present study (data not shown).

Oxidative stress is thought to play a role in the pathogenesis of acute lung injury. ${ }^{47}$ It has been reported that endotoxin treatment results in reduced glutathione levels in the lungs. ${ }^{48}{ }^{49}$ Augmentation of the pulmonary antioxidant status can ameliorate LPS induced lung injury. ${ }^{50}$ MT can also assume the function of superoxide dismutase in yeast ${ }^{51}$ and protect against lipid peroxidation in erythrocyte ghosts produced by xanthine oxidase derived superoxide anion and hydrogen peroxide. ${ }^{52}$ One possibility is that the increased lung inflammation and pulmonary oedema in MT $(-/-)$ mice result from the loss of antioxidative effects caused by MT deficiency. We therefore looked at the contribution of oxidative stress to the aggravation of LPS induced injury in the absence of MT. The immunoreactivity of 8-OhdG, pentosidine, $\mathrm{N}$-(carboxymethyl) lysine (CML), and nitrotyrosine in the lungs of the four experimental groups were compared by immunohistochemistry (data not shown). 8-OhdG is a proper marker of oxidative DNA damage. Advanced glycation end products such as pentosidine and CML are reported to be the final products of oxidative stress. ${ }^{53-55}$ In addition, reactive nitrogen species have a number of inflammatory actions and their products such as nitrotyrosine are accurate biomarkers of oxidation of amino acids. ${ }^{56}$ In the presence of LPS challenge, however, the expression of these molecules did not differ between the two genotypes. Lipid peroxidation is another biomarker for oxidative stress. We have previously reported that lung injury caused by LPS is concomitant with the enhancement of lipid peroxidation in the lung. ${ }^{57}$ We therefore studied the effects of MT on lipid peroxide formation in the lung and found that the lipid peroxide content was higher in the lungs of LPS treated mice than in those of vehicle treated mice, both in the presence and absence of MT. In particular, LPS treatment resulted in significantly higher levels of lipid peroxides in the lungs of MT (-/-) mice than in those of WT mice. It is suggested that the enhancement of lipid peroxidation is involved, at least partly, in the aggravation of acute lung injury induced by LPS in MT $(-/-)$ mice. Lipid peroxidation might also be related to the ultrastructural changes in the endothelial and epithelial cells seen in LPS treated MT (-/-) mice.

In conclusion, this study has shown that endogenous MT protects against acute lung injury induced by bacterial endotoxin possibly via a protective effect on the vascular integrity. The effect does not appear to be mediated through inhibition of the NF- $\mathrm{BB}$ pathway and subsequent proinflammatory cytokine expression. Augmentation of MT may provide alternative therapeutic strategies for acute lung injury, especially when treatments for inhibition of the NF$\kappa \mathrm{B}$ pathway are ineffective.

\footnotetext{
Authors' affiliations

H Takano, K Inove, R Yanagisawa, National Institute for Environmental Studies, Tsukuba, Japan

H Takano, T Yoshikawa, Department of Medicine, Kyoto Prefectural University of Medicine, Kyoto, Japan

M Sato, Department of Hygienics, Gifu Pharmaceutical University, Gifu, Japan
} 
A Shimada, T Morita, M Sawada, K Nakamura, Department of Veterinary Pathology, Faculty of Agriculture, Tottori University, Tottori, Japan

C Sanbongi, Meiji Seika Kaisha Co, Saitama, Japan

\section{REFERENCES}

1 Klaassen CD, Liu J. Metallothionein transgenic and knock-out mouse models in the study of cadmium toxicity. J Toxicol Sci 1998;23:97-102.

2 Klaassen CD, Cagen SZ. Metallothionein as a trap for reactive organic intermediates. Adv Exp Med Biol 1981;136:633-46.

3 Lazo JS, Pitt BR. Metallothioneins and cell death by anticancer drugs. Annu Rev Pharmacol Toxicol 1995;35:635-53.

4 Thornalley PJ, Vasak M. Possible role for metallothionein in protection against radiation-induced oxidative stress. Kinetics and mechanism of its reaction with superoxide and hydroxyl radicals. Biochim Biophys Acta 1985;827:36-44.

5 Abel J, de Ruiter N. Inhibition of hydroxyl-radical generated DNA degradation by metallothionein. Toxicol Lett 1989;47:191-6.

6 Chubatsu LS, Meneghini R. Metallothionein protects DNA from oxidative damage. Biochem J 1993;291:193-8.

7 Schwarz MA, Lazo JS, Yalowich JC, et al. Metallothionein protects against the cytotoxic and DNA-damaging effects of nitric oxide. Proc Natl Acad Sci USA 1995;92:4452-6

8 Bauman JW, Liu J, Liu YP, et al. Increase in metallothionein produced by chemicals that induce oxidative stress. Toxicol Appl Pharmacol 1991:110:347-54.

9 Sato $M$, Bremner I. Oxygen free radicals and metallothionein. Free Radic Biol Med 1993; 14:325-37

10 Takano H, Satoh M, Shimada A, et al. Cytoprotection by metallothionein against gastroduodenal mucosal injury caused by ethanol in mice. Lab Invest 2000;80:371-7.

11 Waelput W, Broekaert D, Vandekerckhove J, et al. A mediator role for metallothionein in tumor necrosis factor-induced lethal shock. J Exp Med $2001: 194: 1617-24$

12 Kimura T, Itoh N, Takehara M, et al. Sensitivity of metallothionein-null mice to LPS/D-galactosamine-induced lethality. Biochem Biophys Res Commun $2001 ; 280: 358-62$.

13 Ware LB, Matthay MA. The acute respiratory distress syndrome. N Engl J Med 2000:342:1334-49.

14 Chollet-Martin S, Montravers P, Gibert C, et al. Subpopulation of hyperresponsive polymorphonuclear neutrophils in patients with adult respiratory distress syndrome. Role of cytokine production. Am Rev Respir Dis 1992:146:990-6.

15 Goodman RB, Strieter RM, Martin DP, et al. Inflammatory cytokines in patients with persistence of the acute respiratory distress syndrome. Am J Respir Crit Care Med 1996; 154:602-11.

16 Suter PM, Suter S, Girardin E, et al. High bronchoalveolar levels of tumor necrosis factor and its inhibitors, interleukin-1, interferon, and elastase, in patients with adult respiratory distress syndrome affer trauma, shock, or sepsis. Am Rev Respir Dis 1992;145:1016-22.

17 Brigham KL, Meyrick B. Endotoxin and lung injury. Am Rev Respir Dis 1986:133:913-27.

18 Hur T, Squibb K, Cosma G, et al. Induction of metallothionein and heme oxygenase in rats after inhalation of endotoxin. J Toxicol Environ Health A 1999;56:183-203.

19 De SK, McMaster MT, Andrews GK. Endotoxin induction of murine metallothionein gene expression. J Biol Chem 1990;265:15267-74.

20 Xing Z, Jordana M, Kirpalani H, et al. Cytokine expression by neutrophils and macrophages in vivo: endotoxin induces tumor necrosis factor-alpha, macrophage inflammatory protein-2, interleukin-1 beta, and interleukin- 6 but not RANTES or transforming growth factor-beta 1 mRNA expression in acute lung inflammation. Am J Respir Cell Mol Biol 1994;10:148-53.

21 Carter AB, Monick MM, Hunninghake GW. Lipopolysaccharide-induced NFkappaB activation and cytokine release in human alveolar macrophages is PKC-independent and TK- and PC-PLC-dependent. Am J Respir Cell Mol Biol 1998:18:384-91.

22 Abdel-Mageed AB, Agrawal KC. Activation of nuclear factor kappaB: potential role in metallothionein-mediated mitogenic response. Cancer Res 1998:58:2335-8.

23 Sakurai A, Hara S, Okano N, et al. Regulatory role of metallothionein in NFkappaB activation. FEBS Lett 1999;455:55-8.

24 Michalska AE, Choo KH. Targeting and germ-line transmission of a null mutation at the metallothionein I and II loci in mouse. Proc Natl Acad Sci USA 1993:90:8088-92.

25 Takano H, Yoshikawa T, Ichinose T, et al. Diesel exhaust particles enhance antigen-induced airway inflammation and local cytokine expression in mice. Am J Respir Crit Care Med 1997; 156:36-42.

26 Takano H, Yanagisawa R, Ichinose T, et al. Diesel exhaust particles enhance lung injury related to bacterial endotoxin through expression of proinflammatory cytokines, chemokines, and intercellular adhesion molecule1. Am J Respir Crit Care Med 2002;165:1329-35.

27 Ichinose T, Furuyama A, Sagai M. Biological effects of diesel exhaust particles (DEP). II. Acute toxicity of DEP introduced into lung by intratracheal instillation. Toxicology 1995;99:153-67.
28 Yanagisawa R, Takano H, Inoue $\mathrm{K}$, et al. Enhancement of acute lung injury related to bacterial endotoxin by components of diesel exhaust particles. Thorax 2003;58:605-612

29 DiSilvestro RA, Cousins RJ. Glucocorticoid independent mediation of interleukin-1 induced changes in serum zinc and liver metallothionein levels. Life Sci 1984:35:2113-8.

30 Cousins RJ, Leinart AS. Tissue-specific regulation of zinc metabolism and metallothionein genes by interleukin 1. FASEB J 1988;2:2884-90.

31 Schroeder JJ, Cousins RJ. Interleukin 6 regulates metallothionein gene expression and zinc metabolism in hepatocyte monolayer cultures. Proc Natl Acad Sci USA 1990;87:3137-41.

32 Sato $M$, Sasaki $M$, Hojo H. Differential induction of metallothionein synthesis by interleukin- 6 and tumor necrosis factor-alpha in rat tissues. Int J Immunopharmacol 1994;16:187-95.

33 Friedman RL, Stark GR. alpha-Interferon-induced transcription of HLA and metallothionein genes containing homologous upstream sequences. Nature 1985;314:637-9

34 Crapo JD. Morphologic changes in pulmonary oxygen toxicity. Annu Rev Physiol 1986:48:721-31.

35 Nelson JM, Duane PG, Rice KL, et al. Cadmium ion-induced alterations of phospholipid metabolism in endothelial cells. Am J Respir Cell Mol Biol 1991; 5:328-36.

36 Courtade M, Carrera G, Paternain JL, et al. Metallothionein expression in human lung and its varying levels after lung transplantation. Toulouse Lung Transplantation Group. Chest 1998;113:371-8.

37 Pitt BR, Schwarz M, Woo ES, et al. Overexpression of metallothionein decreases sensitivity of pulmonary endothelial cells to oxidant injury. Am J Physiol 1997:273:L856-65.

38 Apostolova MD, Chen S, Chakrabarti S, et al. High-glucose-induced metallothionein expression in endothelial cells: an endothelin-mediated mechanism. Am J Physiol Cell Physiol 2001;281:C899-907.

39 Falk S, Goggel R, Heydasch U, et al. Quinolines attenuate PAF-induced pulmonary pressor responses and edema formation. Am J Respir Crit Care Med 1999:160:1734-42.

40 Essler M, Hermann K, Amano M, et al. Pasteurella multocida toxin increases endothelial permeability via Rho kinase and myosin light chain phosphatase. J Immunol 1998;161:5640-6.

41 Blackwell TS, Blackwell TR, Holden EP, et al. In vivo antioxidant treatment suppresses nuclear factor-kappa $B$ activation and neutrophilic lung inflammation. J Immunol 1996;157:1630-7.

42 Shanley TP, Schmal H, Friedl HP, et al. Role of macrophage inflammatory protein-1 alpha (MIP-1 alpha) in acute lung injury in rats. J Immunol 1995: 154:4793-802.

43 Standiford TJ, Kunkel SL, Lukacs NW, et al. Macrophage inflammatory protein-1 alpha mediates lung leukocyte recruitment, lung capillary leak, and early mortality in murine endotoxemia. J Immunol 1995;155:1515-24.

44 Yokoi K, Mukaida N, Harada A, et al. Prevention of endotoxemia-induced acute respiratory distress syndrome-like lung injury in rabbits by a monoclonal antibody to IL-8. Lab Invest 1997;76:375-84.

45 Jones ML, Mulligan MS, Flory CM, Warren JS, et al. Potential role of monocyte chemoattractant protein 1/JE in monocyte/macrophage-dependent IgA immune complex alveolitis in the rat. J Immunol 1992;149:2147-54.

46 Woo ES, Kondo Y, Watkins SC, et al. Nucleophilic distribution of metallothionein in human tumor cells. Exp Cell Res 1996;224:365-71.

47 Gonzalez PK, Zhuang J, Doctrow SR, et al. Role of oxidant stress in the adult respiratory distress syndrome: evaluation of a novel antioxidant strategy in a porcine model of endotoxin-induced acute lung iniury. Shock 1996:6:S23-6.

48 Suntres ZE, Shek PN. Treatment of LPS-induced tissue injury: role of liposomal antioxidants. Shock 1996;6:S57-64.

49 Liaudet L, Murthy KG, Mabley JG, et al. Comparison of inflammation, organ damage, and oxidant stress induced by Salmonella enterica serovar Muenchen flagellin and serovar Enteritidis lipopolysaccharide. Infect Immun 2002;70:192-8.

50 Suntres ZE, Shek PN. Prophylaxis against lipopolysaccharide-induced acute lung injury by alpha-tocopherol liposomes. Crit Care Med 1998;26:723-9.

51 Tamai KT Gralla EB, Ellerby LM, et al. Yeast and mammalian metallothioneins functionally substitute for yeast copper-zinc superoxide dismutase. Proc Natl Acad Sci USA 1993;90:8013-7.

52 Thomas JP, Bachowski GJ, Girotti AW. Inhibition of cell membrane lipid peroxidation by cadmium- and zinc-metallothioneins. Biochim Biophys Acto 1986:884:448-61.

53 Miyata T, Taneda S, Kawai R, et al. Identification of pentosidine as a native structure for advanced glycation end products in beta-2-microglobulincontaining amyloid fibrils in patients with dialysis-related amyloidosis. Proc Natl Acad Sci USA 1996:93:2353-8.

54 Dunn JA, Patrick JS, Thorpe SR, et al. Oxidation of glycated proteins: agedependent accumulation of $\mathrm{N}$ epsilon-(carboxymethy)) ysine in lens proteins. Biochemistry 1989;28:9464-8.

55 Fu MX, Requena JR, Jenkins AJ, et al. The advanced glycation end product, Nepsilon-(carboxymethyl) lysine, is a product of both lipid peroxidation and glycoxidation reactions. J Biol Chem 1996;271:9982-6.

56 Stadtman ER, Oliver CN. Metal-catalyzed oxidation of proteins. Physiological consequences. J Biol Chem 1991;266:2005-8.

57 Yoshikawa T, Takano H, Takahashi S, et al. Changes in tissue antioxidant enzyme activities and lipid peroxides in endotoxin-induced multiple organ failure. Circ Shock 1994;42:53-8. 